\title{
Propagación vegetativa por esquejes de Monttea chilensis Gay
}

\section{Vegetative propagation by cuttings of Monttea chilensis Gay}

\author{
Gabriela Saldías \\ Universidad Central de Chile, Facultad de Arquitectura, Urbanismo y Paisaje, Escuela de Arquitectura del Paisaje. Santa \\ Isabel 1186, Santiago, Chile. \\ mgsaldiasp@ucentral.cl
}

\begin{abstract}
RESUMEN
Monttea chilensis (Plantaginaceae) es un arbusto endémico de zonas áridas y semiáridas de Chile, clasificado En Peligro de Extinción. Con la finalidad de aportar a la conservación ex situ de esta especie se realizaron ensayos de propagación por esquejes. Se utilizaron esquejes binodales, aplicando ácido indol butírico (AIB), en concentraciones de 0, 1500, 3000, 5000 y 7500 ppm, utilizando 60 esquejes por tratamiento. Se mantuvieron en cama caliente $\left(22^{\circ} \mathrm{C}\right)$ y humedad $80 \%$ regulado con la activación de nebulizadores, bajo túnel plástico. La aplicación de AIB no incrementó el enraizamiento, siendo similar la respuesta del testigo a la dosis de 1500 ppm de AIB, alcanzando un 40\% de enraizamiento. Dosis hormonales superiores e inferiores a $1500 \mathrm{ppm}$ tuvieron un efecto adverso.
\end{abstract}

Palabras clave: Flora amenazada, endemismo chileno de zonas áridas, conservación ex situ.

\begin{abstract}
Monttea chilensis (Plantaginaceae) is an endemic shrub to the arid and semi-arid areas of Chile, classified as an Endangered Species. In order to contribute to the ex situ conservation of the species by cuttings propagation, two experiments were conducted. The cuttings used were binodal, and the hormone indol butyric acid (IBA) was applied in the concentration of $1,500,3,000,5,000$ and 7,500 ppm and a control without hormone. Sixty cuttings per treatment were used rooted under propagation bed conditions with basal heat $\left(22^{\circ} \mathrm{C}\right)$ and $80 \%$ humidity regulated with nebulizers under plastic tunnel. The hormone did not increase rooting, resulting in a similar result ( $40 \%$ of rooting) the control cuttings and those with 1,500 ppm of IBA. Higher and lower IBA concentrations had an adverse effect on rooting.
\end{abstract}

KeYwoRDs: Endangered, Chilean arid endemic species, ex situ conservation.

\section{INTRODUCCIÓN}

Los arbustos leñosos y las cactáceas representan formas de vida peculiares de los ambientes áridos y semiáridos de Chile. Contribuyen significativamente a mantener la integridad física de los suelos evitando o aminorando la erosión, aportan materia orgánica y bajo sus copas existen condiciones más favorables para el reclutamiento de plántulas. Los arbustos son importantes no sólo porque confieren estabilidad estructural al suelo sino porque además contribuyen a mantener una alta biodiversidad al favorecer el reclutamiento de nuevas plantas. La eliminación de la vegetación arbustiva acelera el proceso de erosión y la desertificación, además, se pierden importantes funciones ecosistémicas (Gutiérrez \& Squeo 2004).

Actualmente está ocurriendo una fuerte expansión de la actividad agrícola, forestal e inmobiliaria (León et al. 2003), en particular en la Región de Coquimbo, donde la vegetación presenta un gran deterioro en su cobertura y diversidad, llegando muchas especies a estar en condición Vulnerable o En Peligro. Lo anterior es producto de un proceso de malas prácticas, evidenciado por los expertos desde hace muchos años (Gay 2001). Como respuesta a mejorar la situación se ha elaborado una Estrategia y Plan de Acción de conservación de la biodiversidad para la Región de Coquimbo (CONAMA 2002).

De acuerdo a la "Estrategia Global para la Conservación de las Especies Vegetales" (Chacón et al. 2011) en América Latina queda establecida como meta 8 , lograr que al menos el $75 \%$ de las especies vegetales amenazadas se conserve en colecciones ex situ y al menos el $20 \%$ esté disponible para programas de recuperación y restauración. El mismo 


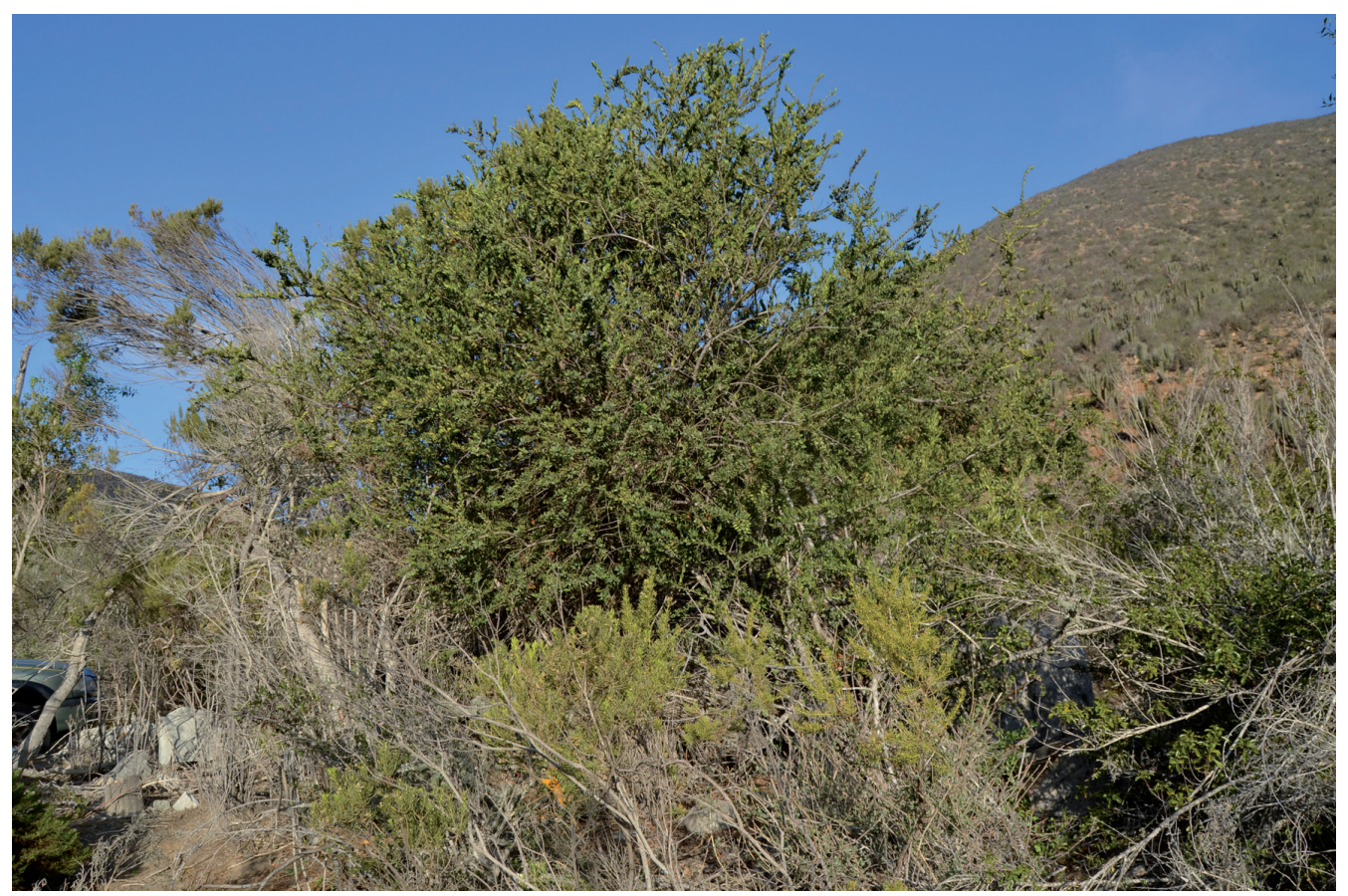

Figura 1. Monttea chilensis en Quebrada El Maray, Región de Coquimbo, Chile.

FIGURE 1. Monttea chilensis in Quebrada El Maray, Region of Coquimbo, Chile.

informe apunta a un programa exhaustivo de conservación ex situ, que complemente la conservación in situ a través del uso de colecciones genéticamente representativas y medidas para mitigar las amenazas al medio silvestre y/o reforzar las respuestas a los posibles impactos del cambio climático. También señala que las colecciones ex situ deberían estar accesibles y respaldadas, ser genéticamente representativas y encontrarse preferiblemente en el país de origen. Para el caso de este estudio acogemos el planteamiento antes descrito por su pertinencia a la delicada situación de deterioro de los paisajes y flora de las zonas áridas y semiáridas del país y las necesidades de disponer de ejemplares bien identificados con fines de rehabilitación, forestación y obras de paisajismo.

El uvillo (Monttea chilensis Gay) pertenece a la familia Plantaginaceae. Es un arbusto siempreverde de copa globosa que alcanza $3 \mathrm{~m}$ de altura por el mismo diámetro (Fig. 1). El follaje es denso, las hojas son coriáceas, las flores pequeñas de color violeta oscuro y el fruto es una cápsula violácea que lleva en su interior una o dos semillas negras y brillantes. Es ornamental por la forma de la copa, el color verde claro del follaje y el contraste que generan los frutos oscuros. Además es visitado por aves que anidan y se refugian entre sus ramas. (Riedemann et al. 2006). No existen antecedentes de usos de la especie y desde el año 1989 se encuentra clasificado como especie amenazada en distintos niveles según diferentes autores.

Es una especie Vulnerable según el Libro Rojo (Benoit
1989); y considerada como En Peligro según Libro Rojo de la Flora de la Región de Coquimbo (Squeo et al. 2001, Squeo et al. 2010). De acuerdo a Gajardo et al. (citado por Benoit, 1989) Monttea chilensis, otrora abundante, ha visto reducida al máximo sus escasas poblaciones y sólo se encontró en una localidad en la Región de Coquimbo. En el tercer proceso de Clasificación de Especies (CONAMA 2008), se clasificó En Peligro debido a que su área de ocupación es inferior a $500 \mathrm{~km}^{2}$, y que esta especie crece en lugares que los agricultores utilizan para plantar parronales. Es una especie endémica de la costa del norte de Chile, donde presenta distribución disyunta con dos núcleos principales en sectores costeros: uno en la Región de Antofagasta, en Paposo entre Miguel Díaz $\left(24^{\circ} 32^{\prime} \mathrm{S}-70^{\circ} 32^{\prime} \mathrm{W}\right)$ y Taltal $\left(25^{\circ} 26^{\prime} \mathrm{S}-70^{\circ} 35^{\prime} \mathrm{W}\right)$, y en la Región de Coquimbo en el Parque Nacional Fray Jorge, en su límite sur, próximo a Los Vilos ( $31^{\circ} 55^{\prime} \mathrm{S}-71^{\circ} 32^{\prime} \mathrm{W}$ ) (Serra et al. 1986). En la Región de Antofagasta habita bajo condiciones de influencia de la neblina costera en una cadena montañosa próxima a la costa. En el sector costero de la Región de Coquimbo, el uvillo se ubica en los sectores bajos de los valles y cuencas, cerca de cursos de agua o napas freáticas accesibles, crece formando matorrales densos de baja altura junto a Schinus polygamus (Cav.) Cabrera. De acuerdo a la clasificación de Gajardo (1994), el uvillo es una especie ocasional del desierto costero de Taltal. También está descrito como parte de la estepa y matorrales costeros de Coquimbo (Serra et al. 1986). 
Se tiene información de reproducción de la especie por semillas (Riedemann et al. 2006), sin embargo, no existen antecedentes de propagación por esquejes.

En este estudio se realizaron ensayos de multiplicación por esquejes, con el objetivo de determinar condiciones para su propagación. La finalidad de la investigación es aportar a la conservación ex situ y fomentar el uso de la especie en obras de paisajismo, recuperación y rehabilitación de paisajes y ambientes degradados, especialmente en su área de distribución.

\section{MATERIALES Y MÉTODOS}

PREPARACIÓN DE ESQUEJES

En septiembre de 2012 se recolectaron ramas de ejemplares adultos de uvillo en la localidad de Quebrada Honda, bosque El Maray, comuna La Higuera, Región de Coquimbo (Fig. 1, 29³4'31,7' S; 71 09'06,6" O, a 543 msnm; 29³4'30,9' $\mathrm{S} ; 7^{\circ} 09^{\prime} 18,5^{\prime}$ ' O, a $\left.519 \mathrm{msnm}\right)$. Las ramas se colocaron en turba húmeda para su transporte en una caja fría y los esquejes fueron preparados dentro de las $48 \mathrm{~h}$ de la recolección. Los esquejes consistieron en secciones binodales, tanto de posición apical como subapicales, y de $5 \mathrm{~cm}$ de longitud aproximada (Delgado et al. 2008, Soto 2004). En todos los casos se eliminaron las hojas inferiores y en la base del esqueje se realizó una lesión longitudinal en la peridermis de aproximadamente $1 \mathrm{~cm}$ para aumentar la superficie de absorción de la hormona (Fig. 2).
De similar manera se prepararon esquejes a partir de brotes obtenidos de ejemplares provenientes de un vivero comercial (Vivero Pumahuida, Huechuraba) y mantenidos en invernadero de la Universidad Central (Santiago, Región Metropolitana) bajo condiciones de riego y fertilidad controladas.

Los ensayos se realizaron en cama de propagación, con calefacción basal $\left(20^{\circ} \mathrm{C}\right)$, bajo túnel plástico, con sistema de control de humedad a $80 \%$, mediante la activación de nebulizadores. Para estimular la formación de raíces se utilizó ácido indol butírico (AIB) en solución alcohólica, a partir de una mezcla comercial (IBA Root Yates ${ }^{\circledR}$ ). Los tratamientos y concentraciones de AIB fueron 1500, 3000, 5000 y $7500 \mathrm{ppm}$. El sustrato de enraizamiento estuvo compuesto por una mezcla en partes iguales de arena gruesa de río, perlita y vermiculita.

Se evaluó la respuesta de enraizamiento a los 52 días de iniciado el proceso, y luego a los 79 y 141 días (Tabla I).

La unidad experimental consistió en 20 esquejes con 3 repeticiones por tratamiento. Las diferencias entre tratamientos fueron evaluadas con la prueba de $\mathrm{X}^{2}$ de Pearson. Para procesar los datos se utilizó el programa Minitab®

Dada la ausencia de respuesta al tratamiento hormonal se diseñó y realizó un segundo experimento con tratamientos en dosis inferiores de la misma hormona (400, 800 y 1500 ppm), pero esta vez utilizando una solución acuosa para evitar un posible efecto fitotóxico por causa del alcohol, asegurando una buena dilución mediante agitación.

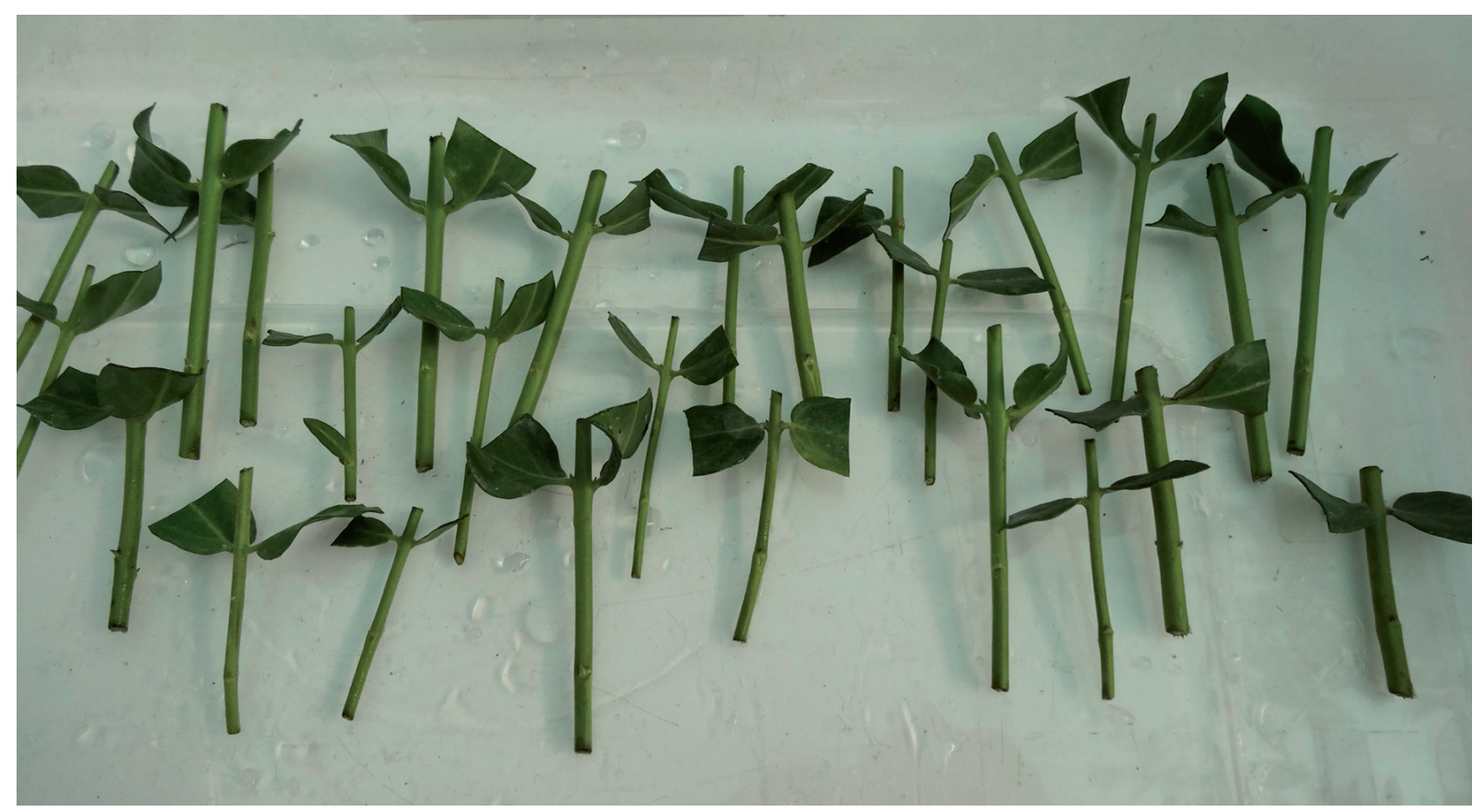

FIGURA 2. Esquejes binodales de Monttea chilensis utilizados para ensayos de enraizamiento.

FIGURE 2. Binodal cuttings of Monttea chilensis used for rooting experiments. 


\section{RESULTADOS}

Los esquejes se secaron paulatinamente sin enraizar. Es importante destacar que el año 2012 fue muy seco en la Región de Coquimbo, con sólo 22,09 mm de precipitación (TuTiempo.net 2016), lo que significó que los tejidos se encontraran muy deshidratados al momento de la corta de ramas.

Se obtuvo enraizamiento en los esquejes preparados con plantas del vivero Pumahuida (obtenidas a partir de semillas) que a diferencia de las muestras silvestres se encontraban bien hidratadas (Tabla I).

El primer registro a los 52 días, el Tratamiento 1500 ppm AIB entregó el mayor enraizamiento de $20 \%$ y el testigo $11,67 \%$. En la evaluación final a los 141 días (aproximadamente 5 meses) el mayor enraizamiento lo alcanzó el testigo (36,7 \%) (Tabla I). No hubo diferencias significativas entre el testigo y $1500 \mathrm{ppm}$.

Concentraciones mayores de AIB (3000, 5000 y 7500 ppm) tuvieron un efecto significativamente inhibitorio en el enraizamiento (Fig. 3).

En la Figura 4A se presentan los esquejes del control registrados a los 79 días, con un promedio de 5,9 raíces por esqueje con un largo promedio de $2,4 \mathrm{~cm}$.

Los resultados del segundo experimento se detallan en la Fig. 5. Por la época del año en que se realizó este experimento (verano-otoño), se trabajó en cama fría con humedad controlada por acción de los nebulizadores bajo túnel plástico.
Concentraciones menores a 1500 ppm de AIB disminuyeron el nivel de enraizamiento. El tratamiento con 1500 ppm de AIB (solución acuosa) alcanzó un 40\% de enraizamiento, siendo el nivel más alto alcanzado en ambos experimentos y entre todas las concentraciones utilizadas.

La cantidad de raíces y el largo promedio en el tratamiento en cama caliente y con la hormona disuelta en solución alcohólica corresponde a 5,9 raíces de un largo promedio de $2,4 \mathrm{~cm}$, a su vez en cama fría y con solución acuosa se obtienen 3,85 raíces por esqueje, largo promedio de 1,63.

Con respecto al crecimiento de los esquejes después de 7 meses desde el enraizamiento alcanzaron un promedio de altura de $19 \mathrm{~cm}$, predominando las plantas con dos tallos $(69 \%)$, en baja proporción con uno (19\%), 3 ó 4 tallos (12\%) (Fig. 4B).

\section{DISCUSIÓN}

Los esquejes preparados a partir de plantas silvestres no presentaron capacidad regenerativa, lo cual sería un reflejo del nivel de estrés hídrico en que se encontraban las plantas. Los esquejes preparados a partir de plantas de vivero enraizaron en cama caliente $\left(22^{\circ} \mathrm{C}\right)$ y en cama fría con humedad controlada (80\%). Esta situación coincide con antecedentes bibliográficos que destacan la importancia de seleccionar material proveniente de plantas sanas y vigorosas, influyendo también la edad de la planta madre y el estado fisiológico (Delgado et al. 2008, Hartmann \& Kester 1999).

TABLA I. Enraizamiento de esquejes de Monttea chilensis mantenidos en cama caliente $\left(22{ }^{\circ} \mathrm{C}\right)$ y humedad alta $(80 \%)$, utilizando distintas concentraciones de AIB en solución alcohólica.

TABLE I. Rooting of cuttings of Monttea chilensis under propagation bed conditions with basal heat $\left(22{ }^{\circ} \mathrm{C}\right)$ and high humidity $(80 \%)$, using different concentration of IBA in alcoholic solution.

\begin{tabular}{|c|c|c|c|}
\hline \multirow{2}{*}{$\begin{array}{l}\text { TRATAMIENTO } \\
\text { (ppm AIB) }\end{array}$} & \multirow{2}{*}{$\begin{array}{c}\text { MOMENTO DE EVALUACIÓN } \\
\text { (días) }\end{array}$} & \multicolumn{2}{|c|}{ ENRAIZAMIENTO ACUMULADO } \\
\hline & & $\left(\mathrm{N}^{\circ}\right)$ & $(\%)$ \\
\hline \multirow[t]{3}{*}{0} & 52 & 7 & 11,7 \\
\hline & 79 & 20 & 21,7 \\
\hline & 141 & 22 & 36,7 \\
\hline \multirow[t]{3}{*}{1500} & 52 & 12 & 20,0 \\
\hline & 79 & 15 & 25,0 \\
\hline & 141 & 16 & 26,7 \\
\hline \multirow[t]{3}{*}{3000} & 52 & 5 & 8,3 \\
\hline & 79 & 10 & 16,7 \\
\hline & 141 & 12 & 20,0 \\
\hline \multirow[t]{3}{*}{5000} & 52 & 6 & 10,0 \\
\hline & 79 & 8 & 13,3 \\
\hline & 141 & 8 & 13,3 \\
\hline \multirow[t]{3}{*}{7500} & 52 & 4 & 6,7 \\
\hline & 79 & 7 & 11,7 \\
\hline & 141 & 8 & 13,3 \\
\hline
\end{tabular}


Propagación vegetativa de Monttea chilensis: SALDÍAS, G.

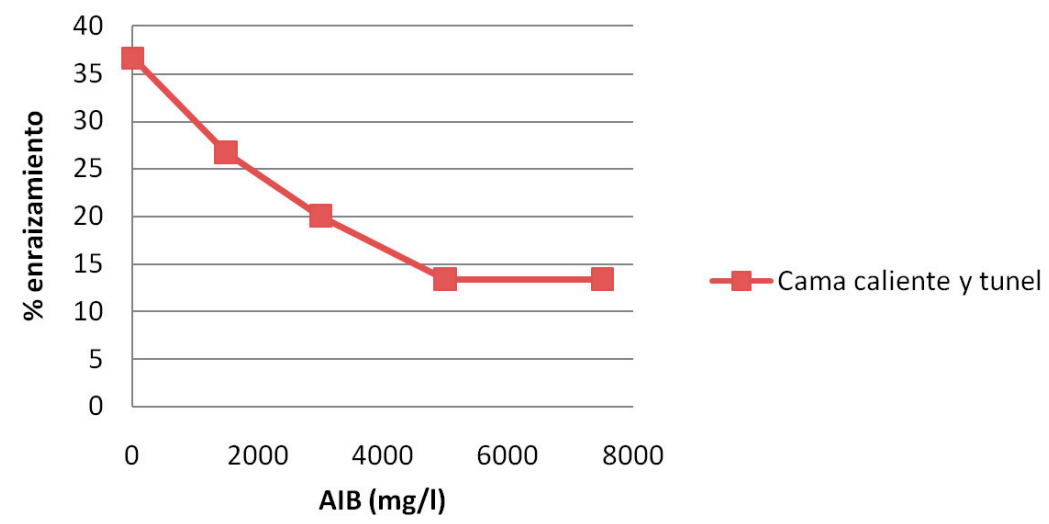

FiguRA 3. Efecto de la concentración de AIB sobre el enraizamiento de esquejes de Monttea chilensis.

FiguRE 3. Effect of concentration of IBA on rooting of cuttings of Monttea chilensis.
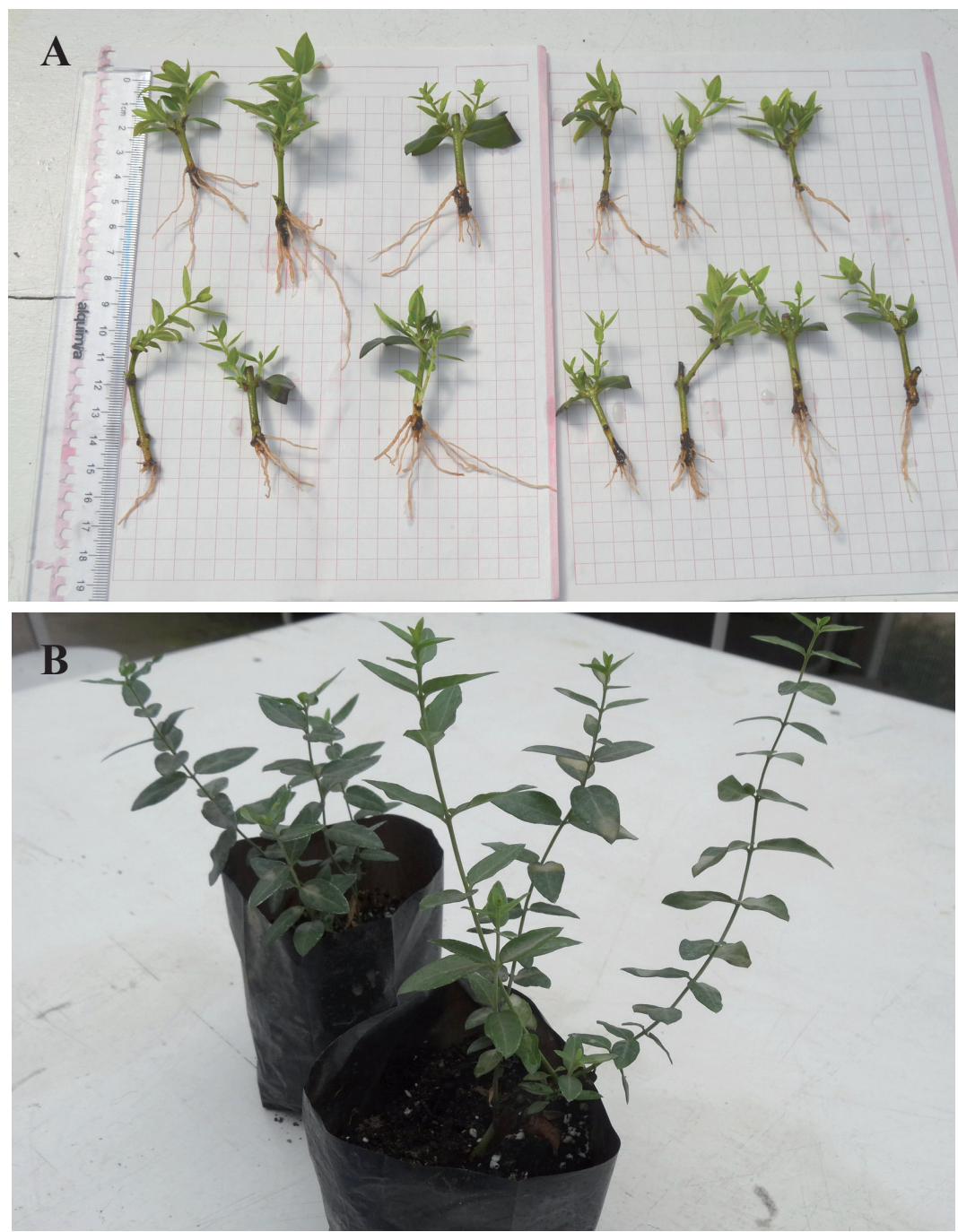

Figura 4. A. Enraizamiento de esquejes de Monttea chilensis del tratamiento testigo (sin AIB), a los 79 días de observación. B. Plantas obtenidas de esquejes, después de 7 meses de enraizamiento.

FiguRE 4. A. Rooting of Monttea chilensis cuttings under control treatment, without hormones, 79 days after the beginning of the experiment. B. Plants grown from cuttings, after seven months of rooting. 


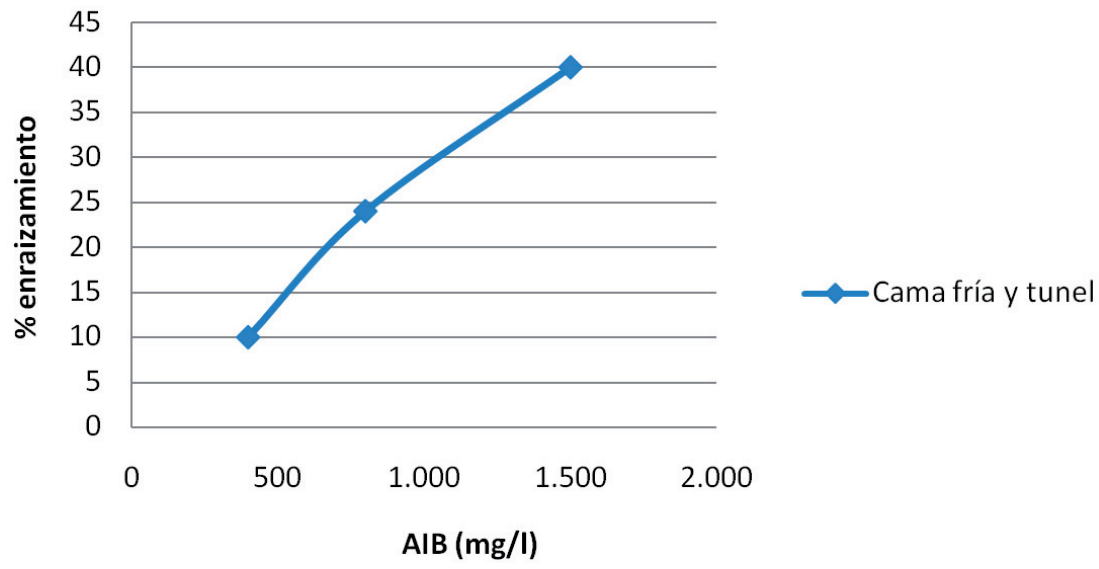

FIgURA 5. Efecto de la concentración de AIB sobre el enraizamiento de esquejes de Monttea chilensis utilizando dosis bajas en solución acuosa.

FIGURE 5. IBA concentration effect on rooting cuttings of Monttea chilensis using low doses in aqueous solution.

Si bien los resultados de ambos experimentos no son comparables porque difieren las condiciones, el máximo de enraizamiento en ambos casos no supera un $40 \%$. La cantidad y largo promedio de raíces es superior cuando la hormona se disuelve en alcohol y se mantienen en cama caliente lo que incide en el establecimiento posterior de las plántulas.

Junto a estos resultados y a su facilidad para generar múltiples tallos a temprana edad, lo convierten en un excelente arbusto para crear cercos o matorrales densos de follaje siempreverde actuando como refugio para la fauna silvestre y cortavista en áreas verdes.

Se conocen otras especies del género Monttea, M. aphylla (Miers) Hauman y M. schickendantzii Griseb. originarias de Argentina, ambas sin antecedentes de su propagación. La familia Plantaginaceae está compuesta principalmente por hierbas, como representantes de la flora nativa está la especie Alonsoa meridionalis (L.f.) Kuntze; sin embargo hay pocos arbustos, entre los que se encuentran especies del género Hebe que se propagan habitualmente por esquejes en los viveros comerciales de plantas ornamentales del país, enraizando fácilmente (Infojardin 2016).

\section{CONCLUSIONES}

El enraizamiento de esquejes de tallos es una forma efectiva de propagación de la especie Monttea chilensis, lo que aporta especial utilidad cuando se desea asegurar las características favorables de ciertos individuos. Es posible alcanzar hasta un $40 \%$ de enraizamiento a partir de esquejes provenientes de plantas bien hidratadas bajo control de humedad $80 \%$, cama fría con dosis de 1.500 ppm de AIB, hormona disuelta en solución acuosa, sin embargo, concentraciones mayores tienen efectos adversos. Resultados similares de enraizamiento $(36,7 \%)$ se logran con tratamiento testigo en cama caliente y usando una solución alcohólica.

\section{AGRADECIMIENTOS}

Se agradece la colaboración del Biólogo Juan Velozo por su aporte en la definición metodológica y a la Ecóloga paisajista Margarita Reyes por su contribución en el trabajo de terreno. Esta investigación fue financiada por el Fondo de Investigación de la Universidad Central de Chile, año 2012-2013.

\section{BIBLIOGRAFÍA}

Benoit, I. (ed.). 1989. Libro Rojo de la Flora terrestre de Chile. Corporación Nacional Forestal. Ministerio de Agricultura, Santiago. 157 pp.

Chacón, P., S. Lagos-Witte, A. Mora \& M. Morales. 2011. Manual para la implementación de la Estrategia Global para la Conservación de las Especies Vegetales (EGCEV) en América Latina. En: https://publicacionesbotanicas. wordpress . com/2014/01/23/manual-para-laimplementacion-de-la-estrategia-global-para-laconservacion-de-las-especies-vegetales-en-americalatina/ Vista: Enero 2016.

CONAMA. Comisión Nacional del Medio ambiente. Estrategia regional y plan de acción de la biodiversidad IV Región de Coquimbo, Disponible en: http://www.sinia.cl/1292/ articles-37022_pdf_estrategia.pdf: Vista: Enero 2016.

Delgado, M., M. Cuba, P. Hechenleitner \& O. Thiers. 2008. 
Propagación vegetativa de Monttea chilensis: SALDÍAs, G.

Propagación vegetativa de taique (Desfontainia spinosa) y tepa (Laureliopsis philippiana) con fines ornamentales. Bosque 29(2): 120-126.

Ficha de Antecedentes de Especie. Id especie 148. http://www. mma.gob.cl/clasificacionespecies/Anexo tercer proceso/ plantas/Monttea_chilensis_FINAL.pdf: Vista Enero 2016

Gajardo, R. 1994. La vegetación natural de Chile. Clasificación y distribución geográfica. Editorial Universitaria. Santiago $165 \mathrm{pp}$.

GaY, C. 2001. Capítulo 19. Sobre las causas de la disminución de los montes de la provincia de Coquimbo. En: F.A. Squeo, G. Arancio \& J.R. Gutiérrez (eds.), Libro Rojo de la Flora Nativa y de los Sitios prioritarios para su conservación: Región de Coquimbo, pp. 281-286. Ediciones Universidad de La Serena, La Serena, Chile.

GutiérRez, J.R. \& F.A. Squeo. 2004. Importancia de los arbustos en los ecosistemas semiáridos de Chile. Ecosistemas 13(1): 36-45.

Hartmann, H. \& D. Kester. 1999. Propagación de plantas: Principios y prácticas. $7^{\mathrm{a}}$ ed. Compañía Editorial Continental S.A. México, DF. 760 pp.

INFOJARDÍN. Hebe Verónica. http://fichas.infojardin.com/arbustos/ hebe-speciosa-hebe-veronica.htm Vista: Enero 2016

León-Lobos, P., H. Way, M. Pritchard, A. Moreira-Muñoz, M. León \& F. CASAdo. 2003. Conservación ex situ de la flora de Chile en banco de semillas. Chloris Chilensis 6(1) En: http://www.chlorischile.cl/bancosemillas/bancosem.htm Vista: Enero 2016.

Riedemann, P., G. Aldunate \& S. Teillier. 2006. Flora nativa de valor ornamental. Zona Norte. Edición 1. Chile. Ediciones JB Chagual, Santiago de Chile. 405 pp.

Serra, M.T., R. Gajardo \& A. Cabello. 1986. Monttea chilensis. Programa de protección y recuperación de la Flora nativa de Chile. Ficha Técnica de especies amenazadas. Corporación Nacional Forestal, Santiago. 10 pp.

Soto, P. 2004. Reproducción vegetativa por estacas en Amomyrtus luma, Amomyrtus meli y Luma apiculata mediante el uso de plantas madres jóvenes y adultas. Tesis para optar al título de Ingeniero Forestal. Universidad Austral de Chile. $64 \mathrm{pp}$.

Squeo, F., G. Arancio \& J. Gutiérrez. 2001. Libro Rojo de la Flora Nativa y de los Sitios Prioritarios para su Conservación: Región de Coquimbo. Ed. Universidad de La Serena, La Serena. $372 \mathrm{pp}$.

Squeo, F.A., C. Estades, N. Bahamonde, L.A. Cavieres, G. Rojas, I. Benoit, E. Parada, A. Fuentes, R. Avilés, A. Palma, R. Solis, S. Guerrero, G. Montenegro \& J.C. Torres-Mura 2010. Revisión de la clasificación de especies en categorías de amenaza en Chile. Revista Chilena de Historia Natural 83: 511-529.

Tu Tiempo. Net. 2016. Clima en La Serena. http://www.tutiempo. net/clima/La_Serena/2012/854880.htm Vista: Enero 2016.

Recibido: 17.08 .15

Aceptado: 09.03.16 